\title{
SIMULAÇÃO E AVALIAÇÃO EXPERIMENTAL DE ESTRUTURAS GEODÉSICAS DE BAMBU REFORÇADAS COM TIRANTES
}

\section{EXPERIMENTAL EVALUATION AND SIMULATION OF GEODESIC STRUCTURES OF BAMBOO RE- INFORCED WITH TIES}

\author{
FABIANO OSTAPIV, Dr. | UTFPR \\ GUSTAVO CORREA | UTFPR \\ JOAMILTON STAHLSCHMIDT, M.SC. | UTFPR \\ GABRIEL OSTAPIV | UTFPR
}

\begin{abstract}
RESUMO
O objetivo deste trabalho é analisar o comportamento de estruturas geodésicas construídas com barras cilíndricas de bambu reforçadas com cabos de nylon por meio de ensaio de carregamento e simulação computacional. Foram modeladas cúpulas geodésicas com forma de um meio icosaedro truncado, construídas com barras comerciais de bambu Phyllostachys pubescens, maciças, com $4 \mathrm{~mm}$ de diâmetro e $175 \mathrm{~mm}$ de comprimento. Foi usado o software de simulação computacional ANSYS para modelar tridimensionalmente e simular a estrutura da cúpula sob ação de carregamentos verticais, com reforço de cabos e também para simular a ação do vento sobre um sólido geodésico. Com a estrutura reforçada, foram feitos carregamentos, para cada carga foi medido o deslocamento vertical da superfície superior em relação à base. O modelo numérico foi simulado com os mesmos carregamentos e, então, foram feitas comparações entre os resultados numéricos e os experimentais. Os ensaios mostram que a estrutura suportou uma carga de 38 vezes o seu peso próprio, apresentando uma grande deflexão sem se romper. Resistiu comparativamente o dobro de carga vertical final com apenas $40 \%$ da deflexão apresentada pela estrutura sem reforço, mostrando a efetividade do uso dos cabos de nylon como reforço amarrado. Tal comportamento havia sido previsto pela simulação numérica. Após realizar os ensaios de carregamento e executar a simulação computacional, foi possível localizar as regiões com as maiores tensões e identificar os possíveis pontos de ruptura da estrutura. Neste trabalho também foi simulado o comportamento da estrutura geodésica revestida por uma casca, sob a ação do vento com diferentes velocidades, mostrando a distribuição da pressão dinâmica exercida pelo escoamento de ar sobre a estrutura.
\end{abstract}

PALAVRAS-CHAVE: Estruturas geodésicas; Barras de bambu; Reforço com cabos; Métodos de Elementos Finitos (MEF)

\begin{abstract}
The objective of this work is to analyze the behavior of geodesic structures built with cylindrical bamboo bars reinforced with nylon cables by means of loading tests and computer simulation. Geodesic domes were modeled in the shape of a half truncated icosahedron and was built with solid commercial bamboo bars Phyllostachys pubescens, with $4 \mathrm{~mm}$ in diameter and $175 \mathrm{~mm}$ in length. ANSYS computer simulation software was used to model three-dimensionally and to simulate a dome structure under the action of vertical loads, with reinforcement of cables and also to simulate a wind action on a solid geodetic. With a reformed structure, loads were made, for each load was measured or vertical displacement of the upper surface in relation to the base. The numerical model was simulated with the same chargers and, afterwards, comparisons were made between the numerical results and the experiments. The tests show
\end{abstract}


that the structure supports a load of 38 times its own weight, presenting a great deflection without breaking. Resistant to comparison or double the final vertical load, with only $40 \%$ deflection. Published by the structure without reinforcement, showing the effectiveness of using nylon cables as tied reinforcement. Such behavior was predicted by numerical simulation. After carrying out the usage tests and running a computer simulation, it was possible to locate the regions with the highest stresses and to identify the possible break points of the structure. In this work it was also simulated or the behavior of the geodesic structure covered by a shell, under a wind action with different movements, showing the distribution of the pressure exerted by the flow of a structure.

KEYWORDS: Geodesic structures; Bamboo bars; Cable reinforcement; Finite elements methods (FEM) 


\section{INTRODUÇÃO}

As cúpulas podem ter grande resistência estrutural se forem usados materiais apropriados e construídas adequadamente. Elas podem cobrir grandes espaços abertos sem a necessidade do uso de suportes internos, além disso, as cúpulas podem ser muito estáveis.

As cúpulas são muito usadas desde a antiguidade na construção de habitações, cobrindo grandes espaços. Os povos originários utilizavam diversos tipos de domos e cúpulas, os índios para construírem suas ocas e os esquimós seus iglus. Van Lengen (2013) mostra muitas destas construções com cúpulas usando arcos, feitas pelos índios da Amazônia, como mostrada na Figura 1.

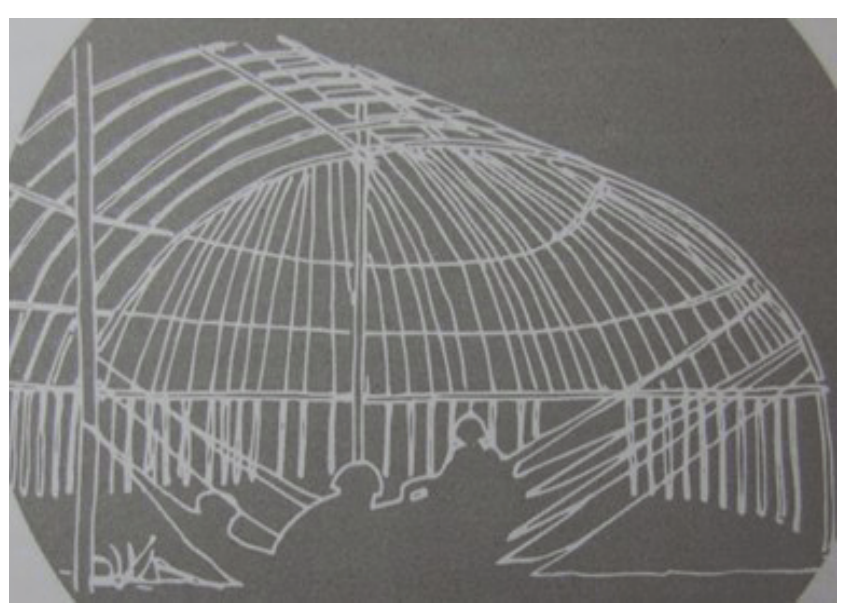

Figura 1 - Desenho mostrando a estrutura de uma habitação indígena em arco. Fonte: Van Lengen, 2013

Geodésicas são estruturas arquitetônicas formadas por arcos, triângulos ou outras formas geométricas regulares que compõem, como uma rede, de uma ou mais camadas, uma superfície espacial curva, geralmente uma semiesfera. Os domos geodésicos podem ser construídos de diversos tamanhos, desde que o comprimento das barras e a frequência das formas geométricas que compõem a superfície da estrutura sejam calculados corretamente.

Geodésicas estão entre as estruturas mais leves e resistentes já inventadas. É um conjunto reticulado, feito geralmente com apenas uma camada, como a geodésica mostrada na Figura 2. Estas estruturas aliam beleza, resistência, leveza, modularidade construtiva, design sustentável e integridade estrutural. Porém, as geodésicas apresentam desafios nas etapas de projeto, execução e no seu uso final, especialmente se for para habitação. Entre estes desafios estão, por exemplo: a seleção de materiais energeticamente eficientes e sustentáveis, o ciclo de vida da estrutura, a repetitividade construtiva e o custo final da obra.

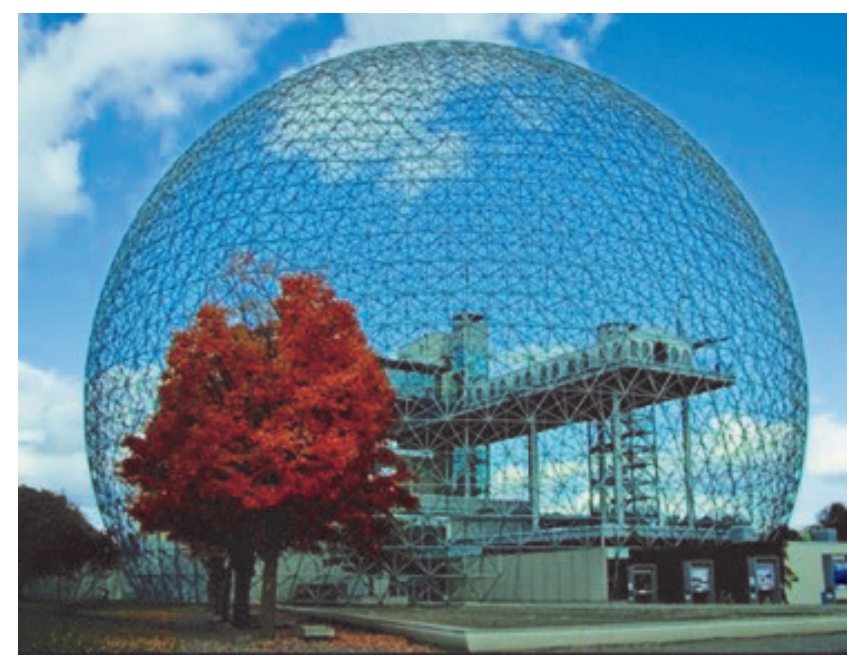

Figura 2 - Cúpula geodésica, biosfera de Montreal no Parque Jean - Drapeau. Fonte: Maia, R.

Colmos de bambus lenhosos podem ser usados na construção de diversos tipos de estruturas de engenharia tais como casas, telhados, pontes, bicicletas, barcos, aviões e também geodésicas. Os colmos de bambu são tubos vegetais segmentados, leves, resistentes e belos. Assim como as madeiras, o bambu é um material lignocelulósico tradicional e confiável que tem baixo peso específico e boa resistência ao carregamento, sendo bastante usado na construção de habitações, principalmente em regiões tropicais onde os bambuzais são abundantes.

O Brasil tem a segunda maior biodiversidade de bambus do mundo, mas apesar de ser facilmente encontrado é uma planta pouco utilizada, devido a vários fatores, tais como: a ausência de políticas públicas de incentivo, o desconhecimento generalizado do seu uso e suas potencialidades, a falta de técnicas e equipamentos para processar este material, a falta de técnicas de construção e preservação adequadas para este tubo vegetal, como relataram Salamon e Ostapiv (2017).

Dependendo da obra, o bambu pode ser processado no local com ferramentas manuais além de possibilitar o envolvimento de trabalhadores locais na construção das habitações. Apesar das dificuldades iniciais em construir com estes tubos vegetais, as construções que usam este material tendem a ser de baixo impacto, baratas, eficientes, rápidas, resistentes e integradas com o meio, conforme apresentaram Librelloto e Ostapiv (2019).

Segundo Ghavami e Moreira (2002), devido ao comportamento de deflexão-compressão apresentado pelos colmos de bambu, este material pode ser usado em diversos tipos de estruturas geométricas, podendo suportar dentro de amplos limites, tanto a carga do vento como carregamentos verticais. Estes autores mostraram que dentro de alguns limites os colmos de bambu podem ser considerados colunas de Euler. 


\section{DOMOS GEODÉSICOS DE BAMBU}

Devido ao tempo de construção de uma cúpula geodésica ser muito curto, quando comparado com outras estruturas de dimensões semelhantes, o uso destas estruturas é indicado para abrigos temporários e de emergência. Domos geodésicos feitos com bambu podem ser usados de inúmeras formas tais como: espaços de exposição em feiras e escolas, acampamentos civis e militares, eventos festivos, espaços temporários para atividades rurais, abrigos para trabalhadores, materiais, animais e equipamentos, etc.

O domo geodésico estudado, desenvolvido por Ostapiv et al (2018). Figura 3a, é uma estrutura tipo icosaedro truncado ou bola de futebol, composta por hexágonos e pentágonos, assim como a estrutura geodésica construída e ensaiada por Castro (2019). Figura 3. Nestas estruturas as barras de bambu usadas têm todas o mesmo comprimento.
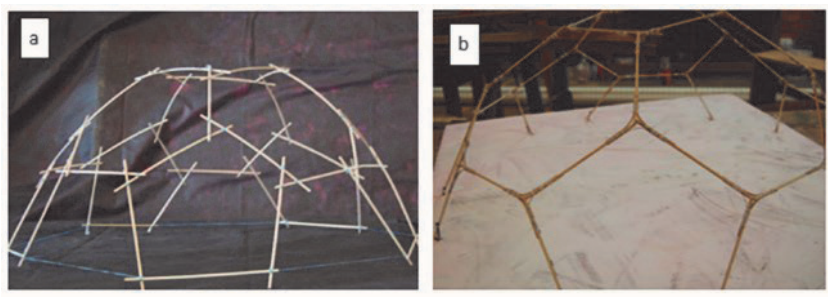

Figura 3 - Domos geodésicos. (a) com varetas de bambu auto apoiadas e amarradas (b) com varetas de bambu amarradas com lâminas de bambu

Fonte: Ostapiv et al (2018), Castro (2019).

Nas estruturas geodésicas, as barras tendem a compartilhar uniformemente as forças de sujeição que são distribuídas de maneira mais ou menos uniforme ao longo de toda a estrutura. Isso faz com que este tipo de estrutura tenha boa resistência a terremotos, por exemplo. Além disso, estruturas com bambu maciço, como a usada na estrutura da Figura 3, têm uma excelente tenacidade, ou seja, uma boa capacidade para absorver impactos.

Domos geodésicos de bambu amarrados podem ser usados também como fôrmas ou estruturas auxiliares na construção de domos de concreto e aço, por exemplo. Podem também servir como andaime para deslocamento dos trabalhadores e como suporte para diversos tipos de materiais como aço, concreto, argila e tijolos, entre outros. Os domos geodésicos de concreto, monolíticos, apresentam uma proteção muito segura contra vendavais, furacões e tornados.

\subsection{Vantagens e desvantagens do uso de do- mos geodésicos}

Por serem esféricas, habitações ou produtos com esta forma apresentam algumas vantagens e também desvantagens em relação às estruturas retangulares tradicionais.
São estruturas versáteis e facilmente adaptáveis às diferentes necessidades do usuário. Suas principais vantagens:

- São estruturas resistentes e boas absorvedoras de vibrações;

- Possuem design único, sem cantos e colunas internas;

- São estruturas bonitas, interessantes e funcionais;

- Tem elevado valor estético e econômico numa construção, agregando valor aos telhados e a obra como um todo;

- As cúpulas geodésicas fechadas são termicamente muito eficientes.

Como as formas esféricas oferecem área superficial mínima para o volume contido por elas, a transferência de calor com o ar externo é a menor possível, pois estas trocas térmicas são diretamente proporcionais à área superficial.

Um dos principais inconvenientes das formas geodésicas em relação às estruturas retangulares tradicionais é a questão da acomodação de peças, acessórios e compartimentos no seu interior, uma vez que estes são normalmente retangulares. Além disso, existem outras desvantagens dos domos geodésicos quando usados para habitações:

- Janelas e portas curvas são mais caras e difíceis de serem encontradas, muitas vezes precisam ser fabricadas sobre encomenda;

- Profissionais que instalam as redes hidráulicas e elétrica normalmente precisam de mais tempo para fazer o serviço que se torna mais caro;

- Sons, cheiros e luz são facilmente difundidos e experimentados em toda a estrutura o que pode causar problemas de privacidade;

- Por ser uma forma radicalmente diferente da tradicional, habitações com este formado podem ser difíceis de vender e apresentam menor valor de mercado.

\subsection{Maquetes de geodésicas}

O uso de maquetes estruturais ajuda no processo de análise, ensaio e simulação, somada a várias outras questões técnicas práticas importantes, que podem ser usadas tanto nas fases de projeto como de execução de uma obra ou de um produto final.

Além de ilustrar o passo a passo construtivo possibilitando a análise modular e a melhoria do processo, o uso de maquetes permite a realização de diversos testes mecânicos que servem, entre outros, para ajustar os parâmetros de modelamento computacional. O comportamento das estruturas em escala menor permite prever o comportamento de estruturas maiores.

Castro (2019) e Ostapiv et al (2019), construíram e ensaiaram maquetes de geodésicas de bambu usando barras cilíndricas maciças de bambu mossô, unidas com resina catalisada 
de poliéster saturada. As barras de bambu foram obtidas a partir de lotes comerciais, de 100 ou 200 unidades, de espetinhos de bambu para churrasco. As barras de bambu utilizadas na maquete têm diâmetro de 4 mm e 175 mm de comprimento. Para fazer a fixação das barras umas com as outras com os ângulos adequados, os autores utilizaram um molde de papelão auxiliar como o mostrado na Figura 4, obtendo uma estrutura cujas características são mostradas na Tabela 1.

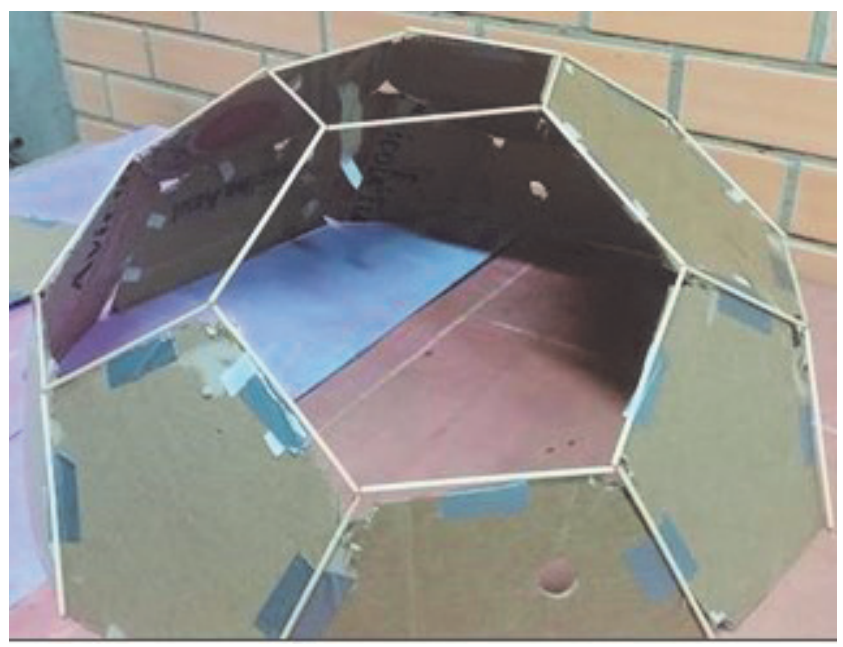

Figura 4-Molde de papelão na forma de cúpula, usado para construção do domo geodésico de bambu. Fonte: Autores

\begin{tabular}{|l|l|}
\hline DOMO GEODÉSICO - icosaedro truncado & \\
\hline Comprimento das arestas dos polígonos da malha & $175 \mathrm{~mm}$ \\
\hline Diâmetro do domo & $800 \mathrm{~mm}$ \\
\hline Altura do domo & $370 \mathrm{~mm}$ \\
\hline Área aproximada da superfície do domo & $1,5 \mathrm{~m} 2$ \\
\hline Volume coberto pelo domo & $98 \mathrm{I}$ \\
\hline Massa total da estrutura & $142 \mathrm{~g}$ \\
\hline
\end{tabular}

Tabela 1- Características do domo geodésico de barras de bambu com aresta de $175 \mathrm{~mm}$. Fonte: Autores

\subsection{Ensaios das cúpulas geodésicas de bambu}

Após a construção as maquetes foram ensaiadas usando carregamento vertical sobre a estrutura para verificar a resistência e a deflexão das estruturas construídas, similar ao trabalho desenvolvido por Ostapiv et al (2018), no qual os autores concluíram que domos geodésicos de bambu amarrados são resistentes, pois, em ensaios a estrutura suportou uma carga de 38 vezes o seu peso próprio, apresentando uma grande deflexão sem se romper.

\subsection{Simulação computacional - Análise por Elementos Finitos}

Cada vez mais são utilizados métodos dos elementos finitos (MEF) para análise de problemas estruturais de engenharia.
Na maioria das vezes, é impossível a obtenção de soluções analíticas dos complexos problemas estruturais existentes na engenharia, daí a importância do uso do método.

O MEF é uma ferramenta altamente eficaz para a obtenção dos campos de deformações e tensões permitindo a identificação das regiões mais solicitadas de uma estrutura. $A$ partir dos resultados da análise numérica, é possível obter um entendimento melhor do comportamento das estruturas, propor e avaliar modificações para otimizar estas estruturas. Castro e Ostapiv et al (2019) usaram o MEF para simular o comportamento de maquetes construídas com palitos de bambu e diferentes tipos de uniões. Para tal análise, usaram os recursos gráficos do programa ANSYS, mostrado nas Figuras 5,6 e 7. Na resolução numérica obtida pelos autores foram considerados grandes deslocamentos dos nós da malha.

Para obter a solução numérica, apresentadas na Figura 5, Ostapiv et al (2019), discretizaram o modelo da geodésica com uma malha de 350 elementos de viga e 695 nós. As condições de contorno usadas no modelamento da geodésica foram: deslocamento e rotação nulos para os nós da base (condição de engaste) e força vertical definida e distribuída nos cinco nós do plano de topo da estrutura. No mesmo trabalho os autores obtiveram valor médio de 12 GPa para o (MOE) módulo de elasticidade das barras de bambu mossô, semelhante ao resultado obtido por Berndsen et al (2013). Este valor do MOE foi utilizado no software ANSYS para a simulação computacional.

Na Figura 5 é mostrada a estrutura inicialmente sem o carregamento e na sequência a Figura 6 mostra a mesma estrutura defletida sob a ação de uma força vertical de $34,06 \mathrm{~N}$ e os respectivos modelos tridimensionais gerados pelo programa computacional.

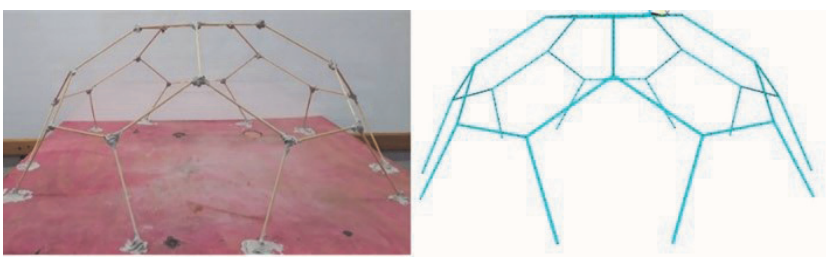

Figura 5 - Estrutura geodésica de barras de bambu sem carregamento eseu modelo tridimensional. Fonte: Autores

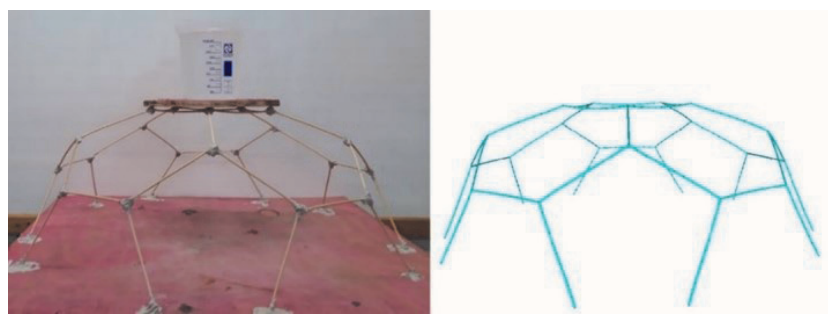

Figura 6 - Geodésica com carregamento vertical e modelo tridimensional nas mesmas condições. Fonte: Autores 
Ostapiv et al (2019) verificaram que as uniões entre os hexágonos da estrutura geodésica são os locais de maior concentração de tensões do conjunto estrutural com o carregamento vertical. A distribuição de tensão equivalente de Von Mises e a tensão máxima de 63,4 MPa são mostradas na Figura 7, no detalhe o ponto de maior tensão na estrutura carregada.

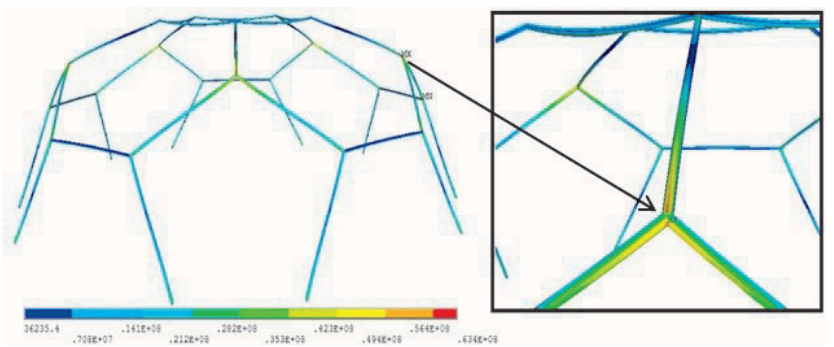

Figura 7 - Distribuição de tensão equivalente de Von Mises. Fonte: Autores

O gráfico 1 mostra a intensidade da força [N] colocada sobre a geodésica e o respectivo deslocamento $[\mathrm{mm}]$ do plano superior desta estrutura. A curva no gráfico mostra a similaridade dos resultados experimentais com os computacionais, obtida por Ostapiv et al (2019). Esta aderência dos resultados é muito importante, pois valida o método computacional e este, entre outras vantagens, permite uma ampla faixa de análises estáticas e dinâmicas da estrutura estudada.

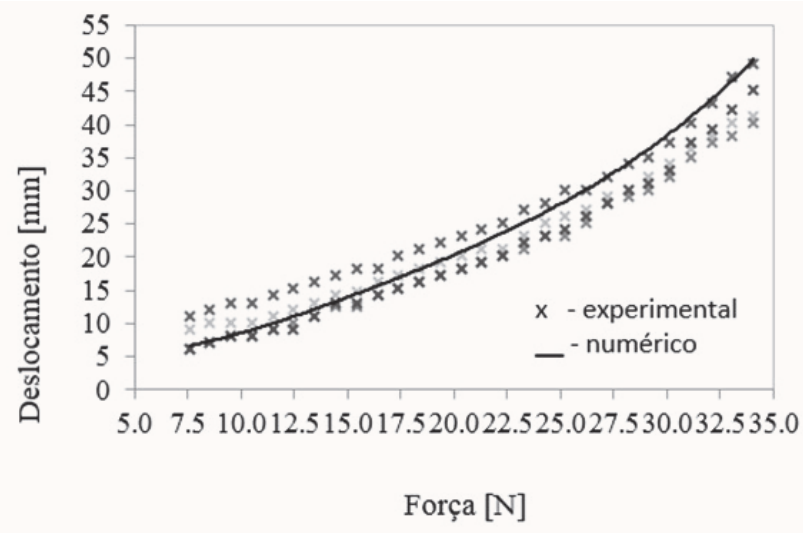

Gráfico 1 - Comparação entre resultados obtidos experimentalmente e por simulação computacional da geodésica de bambu Fonte: Ostapiv et al (2019).

\subsection{Módulo de elasticidade dos cabos de nylon} O módulo de elasticidade (MOE) indica a rigidez de um material. É um indicador macroscópico que tem sua origem na energia de ligação molecular do material quando o material é submetido a um esforço mecânico. $O$ módulo de elasticidade do material é dado pela relação entre a tensão submetida e a deformação ocorrida no material dentro do limite linear-elástico, ou seja, regime no qual o material praticamente não escoa. De um modo geral o nylon, que é o nome comercial para polímeros da família das poliamidas, tem excelente resistência à tração e apresenta MOE entre 1 e $4 \mathrm{GPa}$. O nylon 6.6 é uma fibra sintética, com a qual são feitos os fios trançados ou enrolados de nylon, estes fios podem apresentar valores para o MOE entre 1 e 3,8 GPa.

\section{MATERIAIS E MÉTODOS}

Repetindo o procedimento metodológico adotado por Castro (2019) e Ostapiv et al (2019), para o ensaio da cúpula geodésica de bambu sem reforço, foram construídas maquetes similares as estudadas pelos autores, usando agora reforços de cabos de nylon.

O módulo de elasticidade dos fios de nylon foi obtido a partir do ensaio de tração de 13 corpos de prova, numa máquina universal de ensaios EMIC, no laboratório de materiais da engenharia civil da UTFPR-PB.

Os cabos de nylon usados como reforço foram amarrados em dois planos horizontais distintos da estrutura, ligando os nós (ponto de encontro de 3 barras de bambu) do centro dos hexágonos da estrutura, chamado de plano 1 e logo abaixo na base destes hexágonos, foram amarrados os cabos no plano 2, conforme mostrado na Figura 8 e 9. Estes cabos foram utilizados para atuarem como elementos tracionados e diminuir o esforço sobre esses pontos críticos.

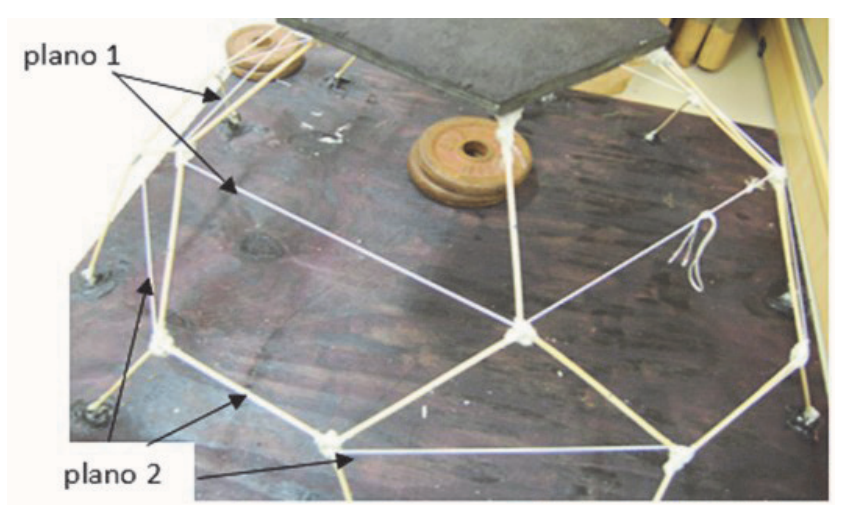

Figura 8 - Detalhes dos planos de amarração dos cabos de nylon na estrutura geodésica de bambu.

Fonte: Autores

Com os valores do módulo de elasticidade das barras de bambu e do cabo de nylon utilizado, foram feitas simulações de carregamento usando o ANSYS, software de desenho e simulação para engenharia. Na Figura 9 é mostrado o modelo numérico utilizado para a simulação computacional com o plano de amarração dos reforços. 


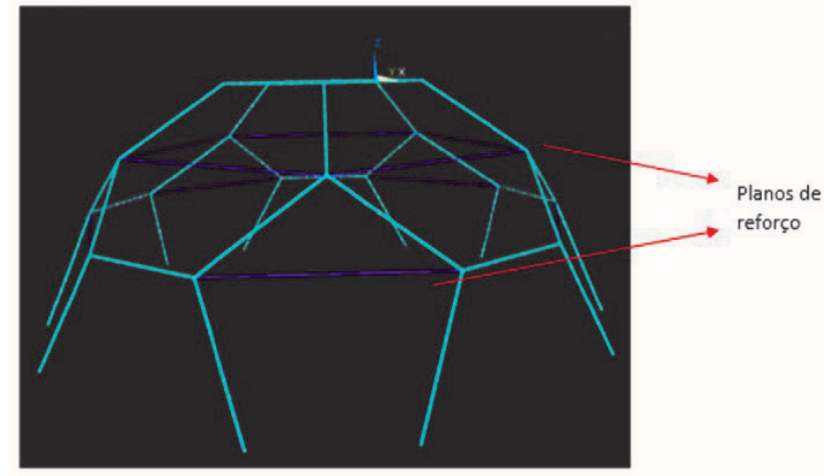

Figura 9 - Desenho da geodésica reforçada no modelo computacional. Fonte: Autores

Com a estrutura reforçada, foram feitos carregamentos utilizando massas de valor conhecido. Para cada carga, foi medido o deslocamento vertical da superfície superior em relação à base. $O$ modelo numérico foi simulado com os mesmos carregamentos e, então, foram feitas comparações entre os resultados numéricos e os experimentais.

Foi utilizado o método dos elementos finitos (MEF) para simular a estrutura. Os palitos de bambu foram modelados utilizando elementos de viga e os fios de nylon como elementos de barra. $\mathrm{O}$ módulo de elasticidade obtido experimentalmente para o fio de nylon foi de 1,25 GPa. Além disso, o fio de nylon possui um diâmetro de 1 $\mathrm{mm}$. A simulação foi feita considerando que os materiais possuem comportamento linear elástico.

A simulação da ação do vento sobre um sólido com a forma da geodésica também foi realizada para entender inicialmente a distribuição de pressão sobre a superfície da geodésica.

\section{RESULTADOS E DISCUSSÕES}

\subsection{Reforço da estrutura usando cabos}

Os valores médios de carregamento e deslocamento, do plano superior da estrutura ensaiada, podem ser observados na Tabela 2.

\begin{tabular}{|l|l|}
\hline Carga $(\mathrm{N})$ & Deflexão média do plano superior da estrutura $(\mathrm{mm})$ \\
\hline 17,5 & 1,0 \\
\hline 22,5 & 2,5 \\
\hline 27,5 & 4,0 \\
\hline 34,8 & 5,5 \\
\hline 46,6 & 8,0 \\
\hline 54,4 & 10,3 \\
\hline 64,2 & 13,3 \\
\hline
\end{tabular}

Tabela 2 - Valores médios da deflexão da estrutura geodésica reforçada com cabos de nylon. Fonte: Autores
Durante o ensaio de carregamento da estrutura geodésica de bambu com reforço amarrado com cabos de nylon, verificou-se que: diminuíram as tensões nas uniões e nas barras de bambu; diminuíram as deflexões da estrutura; aumentaram significativamente a rigidez e a capacidade da estrutura para suportar carga. Evidenciando a efetividade da solução de reforço adotada.

Na Figura 10 são mostrados os gráficos de (carregamento $x$ deflexão) da parte superior da estrutura com e sem reforço de cabos amarrados e as imagens das estruturas geodésicas no momento de máxima carga na situação próxima do colapso da estrutura.

Percebe-se uma maior deflexão do conjunto sem reforço mostrado na Figura 10A, cuja carga máxima foi de 3572g, em relação à estrutura reforçada com cabos mostrada na Figura 10B, cuja carga máxima foi de $6630 \mathrm{~g}$.

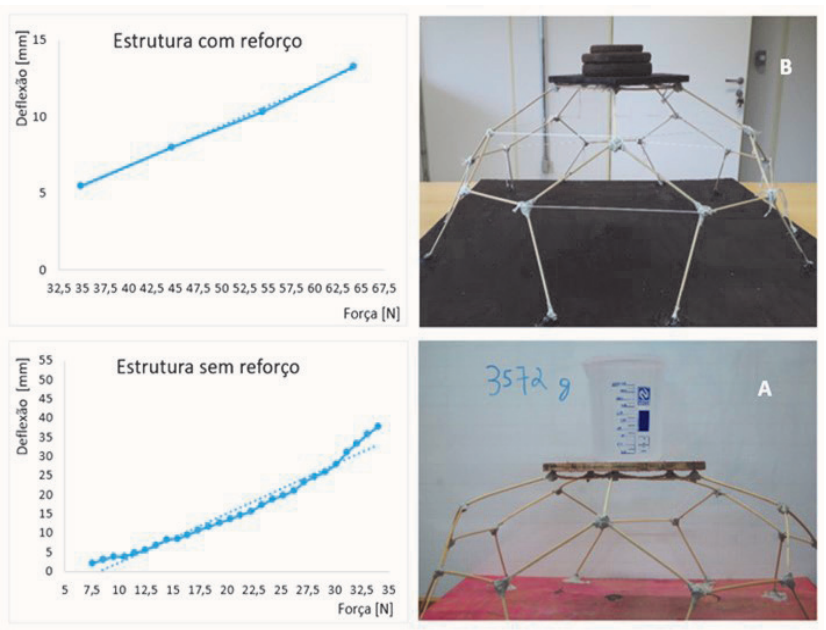

Figura 10 - Curvas de carregamento vertical da estrutura. (A) sem reforço, (B) com reforço. Fonte: Autores

No Gráfico 2 são mostradas as curvas de (carregamento $x$ deflexão) das estruturas com e sem reforço. Comparando estes casos verifica-se que além de resistir ao dobro de carga, a estrutura reforçada defletiu-se muito menos.

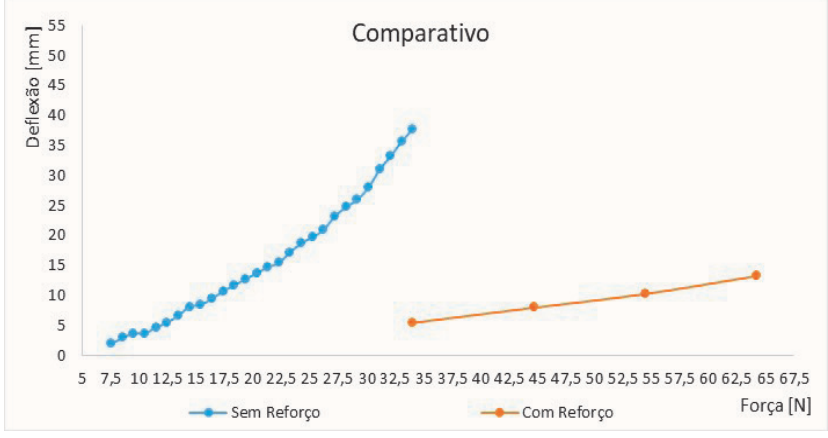

Gráfico 2 - Curvas de carregamento da estrutura geodésica de barras de bambu, unidas com resina, com e sem reforço com cabos de nylon, submetidas a carregamento vertical. Fonte: Autores 
Enquanto a geodésica sem reforço de cabos resistiu uma carga de 23 vezes o seu peso próprio com uma grande deformação vertical do topo da estrutura de $3,2 \mathrm{~cm}$. A geodésica análoga, reforçada com cabos de nylon amarrados, resistiu 4 vezes mais carga para a mesma deflexão da estrutura $(1 \mathrm{~mm})$ e deformou-se oito vezes menos sob a mesma carga $(32,5 \mathrm{~N})$. Nas condições de máximo carregamento a estrutura reforçada resistiu $65 \mathrm{~N}$, ou seja, 42 vezes seu peso próprio, mantendo um comportamento linear de deformação da estrutura como um todo.

\subsection{Simulação estática da estrutura geodésica reforçada}

Após realizar os ensaios de carregamento e executar a simulação computacional para carregamento vertical, foi possível localizar as regiões com as maiores tensões e identificar os possíveis pontos de ruptura da estrutura. Como previsto no modelo numérico, e observado posteriormente no ensaio destrutivo, o ponto de ruptura foi localizado na conexão entre os palitos de bambu da região superior da estrutura.

A Figura 11 mostra o gráfico dos resultados de deslocamento do plano superior da estrutura causada pela força vertical de sujeição. A dispersão dos resultados experimentais se deve basicamente ao grande incremento de carga a cada etapa de carregamento, porém, o modelo numérico consegue se aproximar bem do comportamento médio dos resultados experimentais.

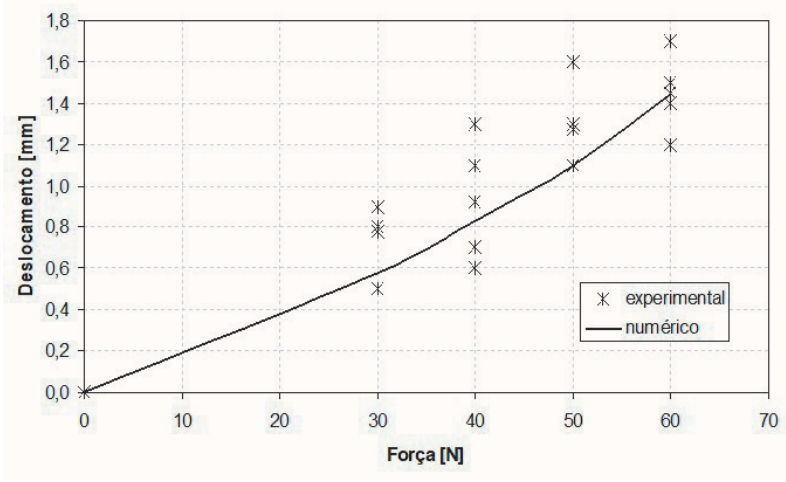

Figura 11 - Deslocamento da estrutura por força de carregamento vertical. Fonte: Autores

O ensaio não destrutivo foi realizado com uma carga máxima de $65 \mathrm{~N}$. O modelo foi simulado com carga máxima de $60 \mathrm{~N}$ e a distribuição da tensão equivalente de Von Mises está mostrada na Figura 12. É possível observar que a tensão máxima de $24,2 \mathrm{MPa}$ ocorre nos fios de nylon do plano superior da estrutura (linhas em vermelho). Desta forma, foi possível aliviar as tensões existentes nas barras de bambu e nas conexões, como mostrado no detalhe da Figura 7, transmitindo parte significativa destas tensões para os cabos tracionados.

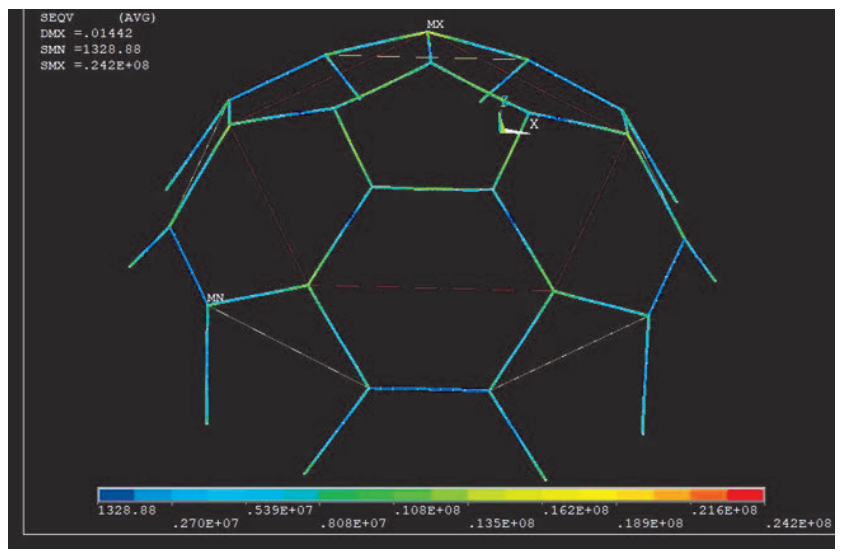

Figura 12 - Distribuição de tensões de von Mises.

Fonte: Autores

O uso do reforço amarrado, na posição adotada foi considerado efetivo, pois permitiu aumentar a resistência mecânica final da estrutura e diminuir sua deflexão, sem agregar peso significativo para o conjunto.

Com o modelo computacional validado, foi possível ampliar a análise através de novas simulações e deste modo predizer o comportamento e a carga máxima suportada pela estrutura. Na Figura 13 é mostrado, através do gráfico de força versus deslocamento, o comportamento da estrutura que foi simulada até o colapso elástico (snap through) a $85 \mathrm{~N}$.

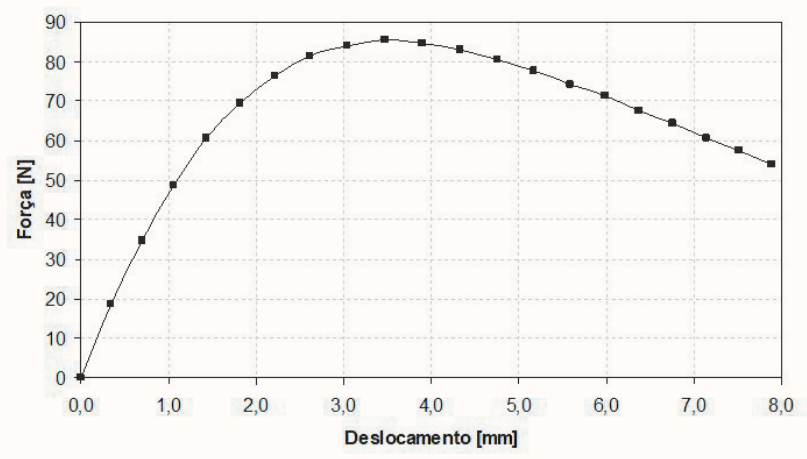

Figura 13 - Força por deslocamento segundo o modelo numérico adotado. Fonte: Autores

A Figura 14 mostra os resultados de simulação do modelo após o colapso elástico da estrutura. As maiores tensões foram agora obtidas nas barras superiores da estrutura. Essas barras também sofreram uma grande deflexão. 


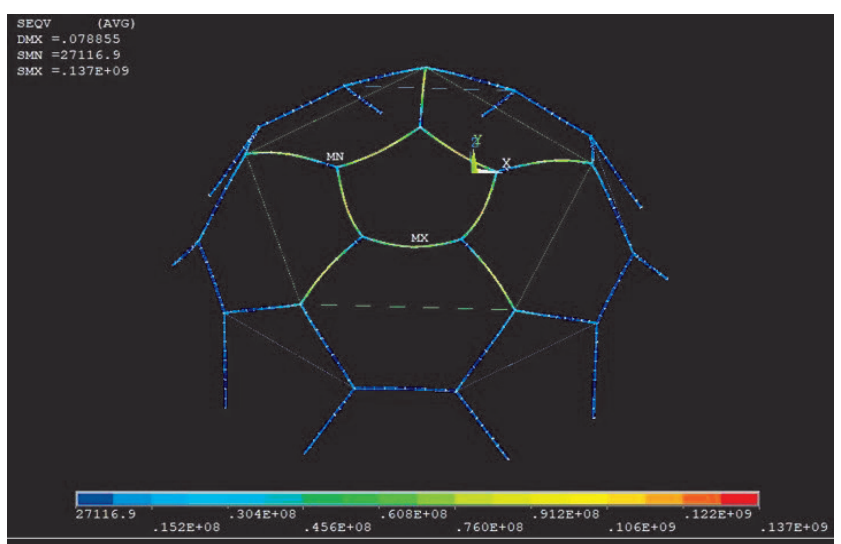

Figura 14 - Colapso elástico do modelo da geodésica. Fonte: Autores

No experimento destrutivo da estrutura geodésica, a carga máxima obtida foi em torno de 72 N. Porém, após atingir a carga máxima, diferentemente do modelo numérico, a estrutura se rompeu na região das conexões entre as barras de bambu.

Na Figura 15 é mostrada a maquete estrutural, instantes antes de ocorrer sua ruptura por carregamento hidráulico. É possível ver as grandes deflexões das barras de bambu que ocorrem especialmente na região superior da estrutura, similares as previstas pelo modelo computacional, mostradas no plano de topo da geodésica da Figura 14.
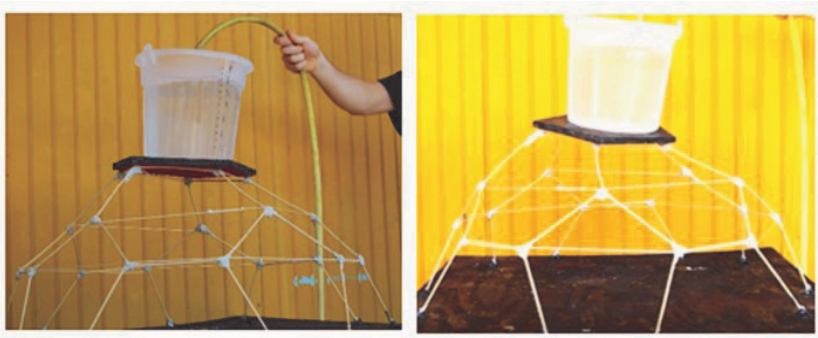

Figura 15 - Estruturas geodésicas de bambu com reforço amarrado próximas do momento de colapso.

Fonte: Autores

Devido às condições geradas pelo carregamento na estrutura, a deflexão da mesma não ocorreu de forma simétrica. Finalmente, a ruptura ocorreu simultaneamente em uma conexão entre as barras, mostrado no detalhe da Figura 16 e também em uma barra de bambu de menor densidade. É preciso ressaltar que o modelo numérico não leva em consideração as propriedades da resina usada nas conexões e por isso não é capaz de predizer a falha frágil deste material.

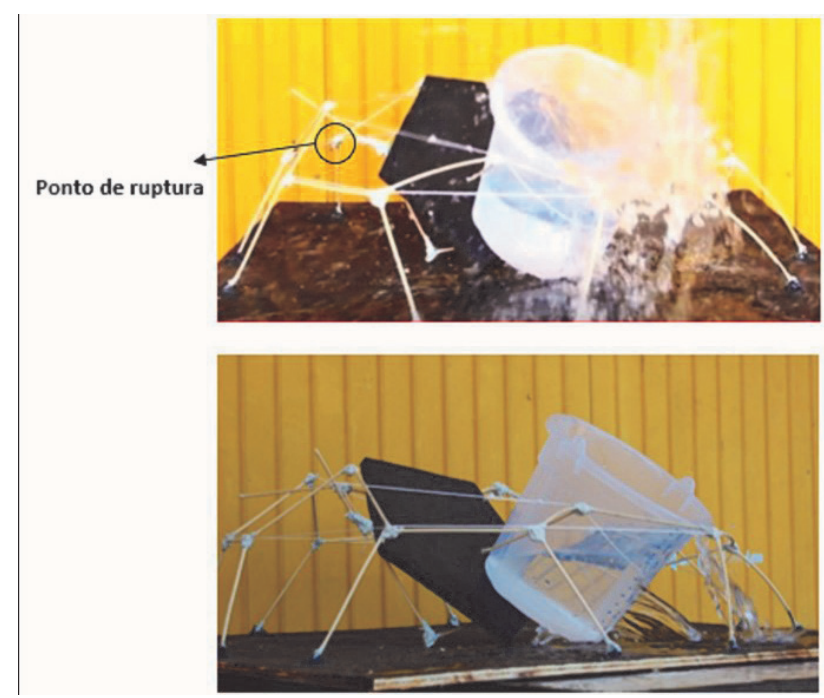

Figura 16 - Colapso elástico da geodésica reforçada no momento do rompimento da estrutura. Fonte: Autores

A carga de rompimento foi de, aproximadamente $72 \mathrm{~N}$. A diferença entre o valor previsto de $85 \mathrm{~N}$ para ruptura e o valor de $72 \mathrm{~N}$ encontrado no ensaio da estrutura pode ser explicado pela seleção inadequada das barras de bambu usadas na construção da estrutura. As mesmas deveriam ter sido classificadas segundo a densidade do material. Algumas destas barras de bambu menos densas têm menor valor de MOE.

Outras fontes de erros podem ser devidas. O desvio do paralelismo do plano superior da estrutura em relação ao plano da base, ocorrida durante o processo de montagem da estrutura. E as diferentes cargas iniciais aplicadas na geodésica no momento de amarração dos reforços, uma vez que o pré-tensionamento dos cabos na amarração foram realizadas manualmente e sem um controle adequado.

Um aspecto importante foi o modo com o qual as estruturas geodésicas com e sem reforço se deformaram. A estrutura sem reforço, a partir do carregamento vertical de $25 \mathrm{~N}$, passou a defletir numa taxa elevada, mostrando que a estrutura se aproxima rapidamente do ponto de colapso (snap through). Por outro lado, a estrutura reforçada com cabos amarrados de nylon, mostrou maior rigidez, maior capacidade de carga e um comportamento de deformação estrutural praticamente linear quando carregada, característica desejável para este tipo de estrutura.

\subsection{Simulação da ação do vento sobre a geodésica}

A simulação de escoamento de ar a $20 \mathrm{~m} / \mathrm{s}$ sobre a geodésica estudada gerou o gradiente de pressão mostrado na Figura 17. Nesta análise a maior pressão dinâmica, de $275 \mathrm{~Pa}$, ocorre no ponto frontal de incidência do escoamento na estrutura, como é mostrado pelas linhas de fluxo da Figura 18. 


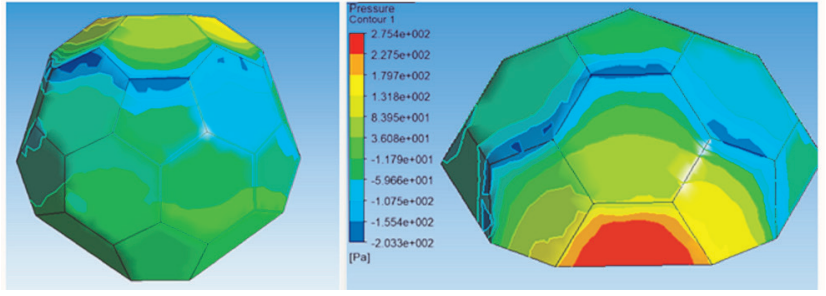

Figura 17 - Distribuição de pressão sobre a superfície geodésica causada pela ação do vento a $20 \mathrm{~m} / \mathrm{s}$.

Fonte: Autores

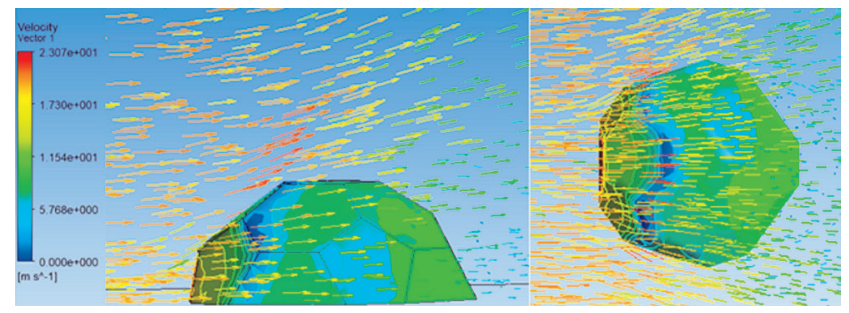

Figura 18 - Linhas de fluxo do vento e velocidade do ar sobre a superfície geodésica. Fonte: Autores

Após incidir frontalmente sobre a geodésica a velocidade do ar aumenta, particularmente na região superior da estrutura, produzindo aí uma zona de baixa pressão e uma força ascendente sobre a geodésica, semelhante ao efeito do escoamento de ar sobre uma asa.

\section{CONCLUSÕES}

Neste trabalho, foram apresentados estudos de simulação numérica computacional de carregamento vertical e escoamento de ar sobre cúpulas geodésicas de bambu reforçadas com amarração. E também ensaios experimentais de carregamento em geodésicas de bambu, construídas em escala reduzida. A união entre as barras de bambu foi feita apenas com resina comercial chamada de "massa plástica", solução já analisada por Ostapiv et al (2019), a partir da solução das uniões das barras, a construção das cúpulas geodésicas se tornou relativamente simples, podendo, no entanto, ser melhorada.

Aplicando o reforço com cabos a geodésica mostrou um comportamento mecânico melhorado. A estrutura resistiu uma carga final cerca de quatro vezes mais elevada e se deformou três vezes menos do que a mesma estrutura sem reforço, aumentando significativamente a rigidez do conjunto. Estes resultados mostraram a importância do reforço e a efetividade da solução adotada que amplia as possibilidades de uso deste tipo de estrutura em telhados, abrigos de emergência e habitações temporárias, por exemplo.

Como discutiram Cook et al (1988), o uso de Métodos de Elementos Finitos MEF, permitiu prever o comportamento da estrutura e testar diferentes soluções de engenharia sobre as mesmas, incluindo aí uma breve análise da ação do vento sobre a geodésica.

No futuro, novas soluções para as geodésicas de bambu podem ser propostas e testadas usando estas importantes ferramentas para a engenharia de estruturas, a simulação computacional e sua validação pelo desenvolvimento e ensaio de modelos físicos em escala reduzida, como os realizados neste trabalho.

\section{REFERÊNCIAS}

BERNDSEN, R.S., KLITZKE, R.J., BATISTA, D.C., NASCIMENTO, E.M., OSTAPIV, F. "Resistência à flexão estática e à compressão paralela do bambu-mossô (Phyllostachys pubescens)" Floresta, v.43, n³, p. 485 494, jul./set. 2013 - Curitiba PR.

CASTRO, G. C. "Ensaio e simulação computacional de estrutura geodésica tipo icosaedro truncado construída com barras de bambu". 93f. 2019. Trabalho de Conclusão de Curso (Graduação em Engenharia Mecânica) - Universidade Tecnológica Federal do Paraná. Pato Branco, 2019.

COOK, R.D., MALKUS, D. S. PLESHA. M. E. "Concepts and Applications of Finite Element Analysis" 3ed. Madison: Editora John Wiley and Sons Ltd, 1988.

GHAVAMI, K., MOREIRA, L.E. "Double-layer bamboo space structures": Space Structures 4, ed: Thomas Telford Ltd, vol.1, p. 573-581. 1993.

HIBBELER, R. C. "Resistência dos materiais". PearsonPrentice Hall, 5 a ed. 670 p., New York, 2010.

HILL, J.O. "Professional Dome Plans", 3a ed. 2002.

VAN LANGDON D. Disponível em: <https://www.archdaily.com.br/br/796023/classicos-da-arquitetura-biosfera-de-montreal-buckminster-fuller $>$. Acesso em 18, Set., 2018.

LENGEN, J.V. "Arquitetura dos índios da Amazônia" B4 Editores, 119p, São Paulo, 2013.

LIBRELLOTO, L.I., OSTAPIV, F. (org) "Bambu caminhos para o desenvolvimento sustentável no Brasil" $1^{\text {a }}$ ed. Virtuhab, 204p. Florianópolis, 2019.

OSTAPIV, F., SALAMON, C., STAHLSCHMIDT, J., BETT, C. F. "Estudos iniciais para ensaios e construção de cúpulas geodésicas usando colmos de bambu". MIX Sustentável, v.4, n.1, p. 108-116. UFSC, Florianópolis, 2018.

OSTAPIV, F., OSTAPIV, G., CASTRO, G.C., STAHLSCHMIDT, J., "Computational simulation and vertical loading tests in bamboo geodesic domes". In: 25th International Congress of Mechanical Engineering ABCM, Uberlândia, MG, out. 2019. 
ROZESTRATEN, A. S. "Apuntes acerca del papel de la representación en el processo del proyecto de arquitectura de Paulo Mendes da Rocha". Arquiteturarevista - Vol. 5, n²: p.111-121. São Paulo, 2009.

SALAMON, C., OSTAPIV, F. Planificação de Colmos de Bambu para Produção de Painéis. Mix Sustentável Edição 05 v.3, n.1. p.73-83. UFSC, Florianópolis, 2017. SALCIDO, J.C.; RAHEEM, A.A.; RAVI, S. "Comparison of embodied energy and environmental impact of alternative materials used in reticulated dome construction" Building and Environment 96: The International Journal of Building Science and its Applications, Elsevier, p. 22-34. 2016.

\section{AUTORES}

ORCID: 0000-0001-5397-1968

FABIANO OSTAPIV, Dr. | Universidade Tecnológica Federal do Paraná | Departamento de Mecânica | Pato Branco, PR Brasil | Correspondência para: Av. Conhecimento Km 1, Bairro Fraron, Pato Branco - PR, 85503-390 | e-mail: fabianoostapiv@utfpr.edu.br

ORCID 0000-0001-5220-461X

GUSTAVO CORREA | Rua Itacolomi 620, Apto 704, Centro, Pato Branco, PR, 85501-240

ORCID: 0000-0003-2594-2187

JOAMÍLTON STAHLSCHMIDT, MSc. | UTFPR, Engenharia Mecânica, Pato Branco, Paraná - Brasil | Rua Arnaldo Moreira Douat, No 150, Ap. 503, Bloco A, Joinville - SC, 89211-000 | e-mail: joamiltons@utfpr.edu.br

ORCID: 0000-0003-2695-4016

GABRIEL OSTAPIV | Universidade Tecnológica Federal do Paraná | Engenharia Mecânica | Pato Branco, PR - Brasil | Correspondência para: R. Pref. Ivo Tomazoni, 140 - La Salle, Pato Branco - PR, 85505-160|e-mail: gabrielostapiv@ gmail.com

\section{COMO CITAR ESTE ARTIGO}

OSTAPIV, Fabiano; CORREA, Gustavo; STAHLSCHMIDT, Joamílton; OSTAPIV, Gabriel. Simulação e Avaliação Experimental de Estruturas Geodésicas de Bambu Reforçadas com Tirantes. MIX Sustentável, [S.I.], v. 6, n. 3, p. 71-82, jun. 2020. ISSN 24473073. Disponível em:<http://www.nexos.ufsc.br/index.php/mixsustentavel>. Acesso em: dia mês. ano. doi:https://doi. org/10.29183/2447-3073.MIX2020.v6.n3.71-82.

DATA DE ENVI0: $16 / 12 / 2019$

DATA DE ACEITE: 20/03/2020 
